\title{
¿Fragmentación predial o estrategias de subsistencia? Una investigación pionera en San Pedro de Atacama (Chile, II Región, 1961)
}

\author{
Property fragmentation or subsistence strategies? A pioneer research in San Pedro \\ de Atacama (Chile, II Región, 1961)
}

Carlos María Chiappe ${ }^{1}$

\begin{abstract}
RESUMEN
El presente artículo tiene como objeto analizar un informe elevado a la Corporación de Fomento de Chile en 1961. Este se titula Elementos diagnósticos para un plan de desarrollo local en San Pedro de Atacama y sus autores son tres integrantes del Instituto de Geografía de la Universidad de Chile. El análisis tanto contextual como textual me llevará a resaltar sus aportes pioneros al conocimiento sobre la agricultura y asuntos conexos en una zona geográfica y localidad específica: la Subárea Circumpuneña y San Pedro de Atacama. Se plantea que estudios de carácter histórico como el presente son indispensables para reconocer que los avances actuales sobre temas geográfico-sociales del Norte Grande chileno se insertan en una determinada genealogía, la cual es preciso tener en cuenta para identificar tanto los logros como las limitaciones pasadas y presentes, dado lo perfectible del conocimiento científico.
\end{abstract}

Palabras clave: estudios andinos, estudios geográfico-sociales, subárea circumpuneña, política desarrollista.

\begin{abstract}
The purpose of this article is to analyze a report submitted to the Corporación de Fomento de Chile in 1961. This is entitled Elementos diagnósticos para un plan de desarrollo local en San Pedro de Atacama and has as its authors three members of the Instituto de Geografía de la Universidad de Chile. The contextual and textual analysis of it will lead me to highlight its pioneers contributions to knowledge about agriculture and related issues in a specific location and area: the Sub-área Circumpuneña and San Pedro de Atacama. It is argued that studies of historical tone such as the present are indispensable to recognize that the current advances on geographic-social issues of the Norte Grande de Chile are inserted in a certain genealogy, which must be taken into account to identify both the achievements and the past and present limitations, given the perfectibility of scientific knowledge.
\end{abstract}

Key words: andean studies, geographical-social studies, sub-área circumpuneña, development policies.

\section{Introducción}

Las discusiones actuales sobre el hombre y su medio en las tierras áridas del Norte Grande de Chile no deberían ignorar que nuestros conocimientos se insertan en una genealogía a la vez variada (multidisciplinar) y específica (nutrida de paradigmas epocales). Si bien aún queda por saldar la discusión sobre la calidad acumulativa o revolucionaria/ paradigmática (sensu Kuhn, 2005) del conocimiento científico, es innegable que cualquier nuevo aporte no se realiza desde el vacío, sino que tiene antecedentes que es bueno no desconocer, porque nos permiten entender mejor el camino que lleva desde las elucubraciones pioneras al estado del entendimiento actual. Esto, a su vez, permite realizar una crítica de este último, ya que la perspectiva histórica demuestra que el conocimiento científico siempre es provisional y perfectible, lo cual constituye una de sus características principales.

1 Universidad Católica del Norte, Instituto de Investigaciones Arqueológicas y Antropológicas. Calle Gustavo Le Paige 380, San Pedro de Atacama, Antofagasta, Chile.

* Autor por correspondencia: carlos.chiappe@ucn.cl

Fecha de Recepción: 07 octubre, 2018.

Fecha de Aceptación: 04 diciembre, 2018.

DOI: http://dx.doi.org/10.4067/S0718-34292019005000201. Publicado en línea: 11-junio-2019. 
En los trabajos sobre el desarrollo de los estudios andinos chilenos, las investigaciones sobre la agricultura tienen -por fuerza propia- una presencia destacada (cfr. Chiappe, 2015). Los desafíos que el ambiente del Norte Grande ha presentado a sus pobladores están en la base de la mayoría de las pesquisas, ya sean estas geográficas, arqueológicas, históricas o etnográficas. Siguiendo esta línea, en el presente artículo traeré a colación una investigación realizada en San Pedro de Atacama en 1961 por investigadores del Instituto de Geografía de la Universidad de Chile (Aranda et al., 1968). Esta tiene estrecha relación con publicaciones anteriores de parecido tono (cfr. Chiappe 2018), en tanto nos posiciona de lleno en una problemática de época: la cuestión del desarrollo, que -aunque mantiene plena vigencia- constituía a inicios de la década un tema candente, dado que se estaba a las puertas de la reforma agraria (1962-1973).

Partiendo de los elementos anteriores, desarrollaré el tema propuesto conjugando el análisis del lenguaje con el cual se expresan las ideas, y "las condiciones histórico-sociales, institucionales y materiales, dentro de las cuales -y con las cuales- se producen". (Consejo de Dirección Prismas. 2007: 150). El texto se compone de dos apartados. El primero es de tipo contextual, y tiene como objeto brindar un marco de época sobre el estado de las investigaciones de carácter aplicado en el Norte Grande entre 1960 y 1970. El segundo analiza el informe aludido: Elementos diagnósticos para un plan de desarrollo local en San Pedro de Atacama (Aranda et al., 1968). Las conclusiones llevan a resaltar las bondades y limitaciones del informe, tanto por su calidad pionera como por sus aportes, que van más allá de una tentativa de evaluación diagnóstica sobre el tema agrícola local (Figura 1).

\section{Políticas públicas y estudios andinos sobre el Norte Grande (1960-1970): la problemática del desarrollo}

En este artículo utilizo la categoría estudios andinos en un sentido amplio, inspirado en la forma adoptada por Lautaro Núñez (2013). Es decir, para referirme a un conjunto heteróclito de investigaciones sobre el norte de Chile, que -tras iniciarse a mediados del siglo XIX-fueron cambiando su orientación tanto por el desarrollo de los intereses científicos como de sus contextos de producción (cfr. Chiappe, 2015). En la etapa que abarca el informe (1950-1970), llamada por Núñez (2013) de apertura científica, la institucionalidad de los estudios andinos se basó en

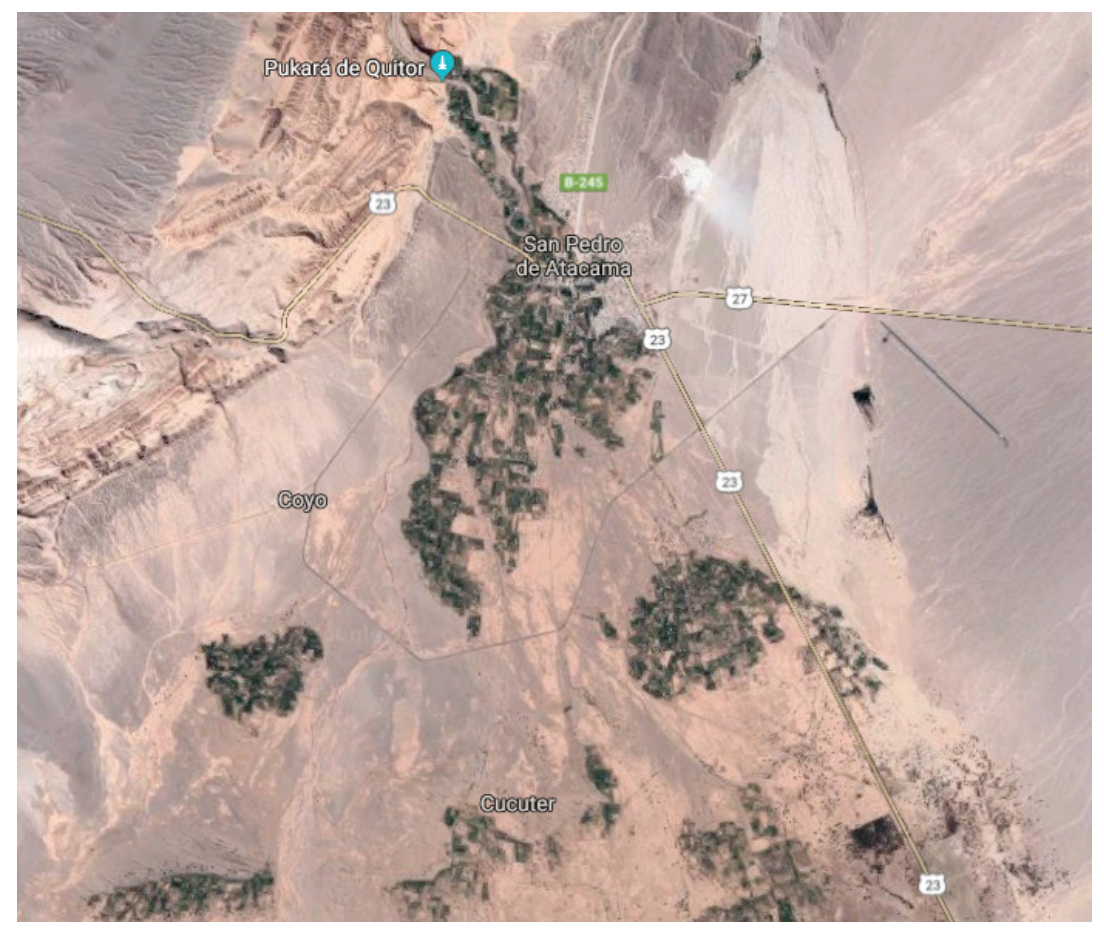

Figura 1. Imagen satelital actual de San Pedro de Atacama y sus ayllus. Fuente: Google Earth 2018 
las dos grandes redes académicas del país: la estatal y laica de la Universidad de Chile, con sede en Santiago y-desde 1960- en Arica, Iquique y Antofagasta, y la privada y católica, representada por la Universidad del Norte, que se fundó en Antofagasta en 1956. Sin embargo, debemos tomar en cuenta también la presencia de diversos museos regionales donde los pioneros locales realizaron tareas de investigación preferentemente arqueológicas (Núñez, 2013). Es el caso de los museos de San Pedro de Atacama (1957) y de Arica (creado en 1959 y transformado en 1967 en el Museo Arqueológico San Miguel de Azapa), los cuales fueron integrados posteriormente a la Universidad del Norte y pertenecen actualmente a la Universidad Católica del Norte y a la Universidad de Tarapacá, respectivamente.

La generación de nuevas estructuras de investigación y docencia, y de medios de comunicación y producción científica, conllevó la incorporación de un conjunto de profesionales que constituyeron el plantel pionero que formaría las próximas generaciones de investigadores locales. Desde la fundación de la Universidad del Norte y la creación de las sedes regionales de la Universidad de Chile se pueden destacar como puntos relevantes el Programa Arqueología y Museos de la Universidad de Chile (1963-1974); la creación del Departamento de Antropología y de la Escuela de Antropología de la Universidad del Norte (1971); el Plan de Desarrollo 1972-1976 de la Universidad del Norte; el Primer Encuentro Arqueológico Internacional de Arica (1961), organizado por el Museo Regional de Arica con el apoyo de la Universidad de Chile y de la Junta de Adelanto de Arica; el Primer Congreso Internacional de Arqueología (1963) organizado por la Universidad del Norte en el Museo de San Pedro de Atacama; el Primer Congreso Nacional del Hombre Andino (1973), organizado por la Universidad de Chile; y el lanzamiento de las revistas Chungara (1972) y Estudios Atacameños (1973), de la Universidad del Norte, hoy universidades de Tarapacá y Católica del Norte, respectivamente. Entre los investigadores pioneros se puede destacar a Gonzalo Ampuero, David Berwick, Sergio Erices, Oscar Espoueys, Liliana Ulloa, Gabriel Martínez, René Lara, Guillermo Focacci, Verónica Cereceda, Héctor Garcés, Nelson Vergara, Juan Munizaga, Patricia Soto, Mario Rivera, Lautaro Núñez, Alfredo Wormald, Bente Bittman, José María Casassas, Tristan Platt, Jorge Hidalgo, Juan van Kessel.

De modo que un rasgo particular de este período es que, aunque la formación de grado de quienes orientaron la investigación social en el norte seguía realizándose en Santiago (particularmente en el Pedagógico de la Universidad de Chile), pues las instituciones nortinas demorarían todavía unos años en generar sus primeras camadas, la investigación dejó de estar originada únicamente en el centro del país. Así comenzó a formarse un importante polo científico en el Norte Grande, que es germen del actual (Universidad Católica del Norte, Universidad de Tarapacá, Universidad de Antofagasta, Universidad Arturo Prat).

Si bien a partir de la década de 1950 puede destacarse un conjunto de investigaciones sociales de interés científico general, lo característico del período empieza a evidenciarse a partir de 1960, cuando el foco se coloca sobre la cuestión del etnodesarrollo en vinculación con el desarrollo regional y nacional. Por lo tanto, lo que me interesa destacar es el carácter predominantemente aplicado de estas ciencias sociales. No significa esto que todas las investigaciones caigan en esta caracterización. Sin embargo, este fue su rasgo más llamativo y la resultante de la convergencia entre el proceso de institucionalización y el momento histórico en el que esta se llevó a cabo, caracterizado por los cambios que conmovieron los cimientos tradicionales del país y que llevaron a implicar a los cientistas en las políticas desarrollistas implementadas por el Estado nacional, en el marco del impulso mundial dado al campo de la investigación y el desarrollo. En este sentido, no resulta casual que la etapa de apertura científica referida por Núñez (2013) haya estado atravesada a la vez por las reformas universitaria y agraria.

La incipiente ciencia social del norte de Chile señalaba por entonces lo pobre de la política estatal orientada a la mejora de los pueblos indígenas de la región. Caben aquí las actuales consideraciones de Lautaro Núñez sobre las razones teórico-prácticas para la realización del Primer Congreso del Hombre Andino (2010, 2013); así como trabajos de época que expresan tanto las visiones culturalistas (Rivera, 1973a; 1973b) como marxistas (Taberna, 1973) sobre la problemática, características de la polaridad política de ese periodo.

El repaso de las políticas públicas implementadas avala este aserto en tanto no hubo hasta mediados de la década de 1960 un programa estatal integrado de desarrollo económico-social. Hasta la presidencia de Frei (1964-1970) sólo destacan:1) la inversión en infraestructura minera y la centrada en las ciudades; 2 ) los proyectos de la Corporación de Fomento (CORFO) para el uso de las aguas subterráneas; y 3) un programa de socorro a pueblos precordilleranos y altiplánicos 
para dotarlos de tecnologías y principios organizativos comunitarios de tipo urbano. Para este período, en resumen, los programas de desarrollo económicosocial fueron escasos, descoordinados entre sí y de una orientación cultural urbana que desnudaba el desconocimiento de la realidad sociocultural de la zona (Pérez Rodríguez, 1984).

La situación cambió durante el gobierno de Frei. Indican Gundermann Kröll y González Cortez (2009) que, a fines de la década de 1960, la profundización de la reforma agraria incidió en la vida de las comunidades indígenas, las que incorporaron el cooperativismo en relación con la tenencia de la tierra, vieron modificada su infraestructura de riego y acumulación, los calendarios agrícolas tradicionales, los sistemas de organización y distribución del agua, e incorporaron también cambios tecnológicos, como nuevos cultivos y maquinaria. Otro elemento que alentó la intervención fueron los informes de la Oficina de Planificación Nacional, que definían al Norte Grande como una región a la cual se debía prestar una mayor atención, ya que su proceso de despoblamiento era grave y repercutía negativamente en la situación geopolítica de la frontera. Esto impulsó a la CORFO a implementar programas que crearon estaciones experimentales orientadas al fomento de la actividad agropecuaria en las pampas, la precordillera y el altiplano, y a ejecutar un plan ganadero basado en auquénidos aportados por la Junta de Adelanto de Arica (1966-1969). En este período comenzaron también las inversiones relativas al campo geotérmico para la producción de energía (Pérez Rodríguez, 1984). Fue este el contexto en el que los entes gubernamentales convocaron a especialistas de las ciencias sociales para asesorarse sobre los alcances y las consecuencias de estas intervenciones (Garretón, 2005).

Durante el gobierno de Salvador Allende (19701973) los planes de desarrollo se reorientaron para exceder lo meramente económico y asentarse en un conocimiento socio-cultural de la región. Esto se basaba en las investigaciones que se habían empezado a realizar alrededor de la problemática aymara. Se dio una colaboración entre universidades y organismos estatales con el objeto de proyectar programas de desarrollo socio-económicos, como fue el caso del interés por acrecentar las factorías de charqui, lo que demuestra mayor conocimiento de la realidad económica y sociocultural del interior de la región (Pérez Rodríguez, 1984). Se debe destacar también la realización de obras públicas por parte de la Junta de Adelanto de Arica (1958-1976), organismo financiado con los impuestos percibidos por el Puerto Libre, que pretendió "erigirse en el modo válido de regionalización, favoreciendo el desarrollo de la industria y convirtiéndose en el motor de la inversión pública”. (Galdames Rosas y Ruz Zagal, 2010).

De modo que el informe a analizar se insertó en este marco y se realizó en el momento inmediatamente previo a la implementación de la reforma agraria. Esta fue expresión paradigmática de todas las grandes transformaciones que encaró el país durante el período de apertura científica. Tanto la reforma como el avance del campo científico se forjaron en el mismo contexto sociopolítico, cuya problemática central giraba en torno a la cuestión del desarrollo. La reforma atacó el régimen latifundista, denotado por las fuerzas que movilizaron el cambio como una antigualla que impedía el desarrollo del país. Y, en esa tarea, los científicos fueron actores activos, realizando estudios sobre el problema agrario que ayudaron a producir conocimientos que sirvieron de base a las políticas implementadas.

\section{Elementos diagnósticos para un plan de desarrollo local en San Pedro de Atacama}

Son varios los trabajos que demuestran la relación entre los estudios andinos y la problemática del desarrollo (cfr. Chiappe, 2015). Sobre el particular, se ha señalado que "los trabajos mejor logrados vienen de geógrafos que estudiaron San Pedro de Atacama por encargo de la CORFO, en función de generar cambios en la agricultura y la sociedad locales". (Gundermann Kröll y González Cortez, 2009: 117). Tomando en cuenta lo anterior, en este apartado examinaré el informe firmado por Ximena Aranda, Rafael Barahona y René Saá (1968) titulado Elementos diagnósticos para un plan de desarrollo local en San Pedro de Atacama (en adelante, el Informe). Este constituye un acabado ejemplo de la colaboración que se dio por esos años entre el gobierno nacional y las universidades, con el fin de diagnosticar los problemas y proponer soluciones para el desarrollo en diferentes regiones del país.

Con respecto a los autores, pertenecían al Instituto de Geografía de la Universidad de Chile (Santiago). En particular sobre Ximena Aranda, Gunderman y González (2009) señalan que se vinculó hacia fines de la década a otros académicos de formación marxista (Lautaro y Patricio Núñez, Freddy Taberna y Gabriel Martínez) interesados en evaluar el papel del campesinado en los cambios revolucionarios. Estos se 
inspiraban en los aportes intelectuales del arqueólogo Julio Montané y del indigenista Alejandro Lipschutz, y tenían también relaciones con otros intelectuales de izquierda de los países vecinos.

David Baytelman, Jefe del Departamento de Desarrollo Agrícola de la CORFO, proponía en la presentación del Informe que los problemas de desarrollo regional se relacionaban con el despoblamiento del Norte Grande como consecuencia de la finalización del ciclo del salitre (ca. 1930) y la imposibilidad actual de implementar una agricultura sostenible. Enfatizaba además que los planes desarrollados se habían concentrado sólo en acceder al recurso de las aguas subterráneas, cosa que -aun habiéndose conseguido- no había solucionado el problema agrícola, porque las condiciones del suelo no eran favorables. Por lo tanto, la realidad del campo consistía en una pobre productividad, una mala utilización de los recursos disponibles y la migración hacia los sectores mineros que ofrecían mejores beneficios, lo que traía aparejada una merma de la población en edad activa en las zonas de emigración.

Lastimosamente, los fracasos de la CORFO contrastaban con la alta productividad que -según las evidencias arqueológicas- había tenido el área en la época precolombina. Para Baytelman, esto se debía a que "los pasos que los organismos estatales daban en el desarrollo de la agricultura en el Norte Grande [se habían hecho] de acuerdo a una forma absolutamente anárquica y tomando aspectos parciales de la realidad regional". En este sentido, el Informe tenía gran relevancia porque por primera vez se abarcaba "el problema agrícola en su conjunto", lográndose determinar "el efecto de los diversos factores que intervienen en el proceso productivo", jerarquizando y priorizando "las variables que se constituyen en limitantes o determinantes del progreso". San Pedro de Atacama fue elegido como lugar de experimentación por ser un poblado de "cierta dimensión" ubicado cerca de los centros de consumo. Allí, la CORFO estaba interesada en entender "cuáles eran los factores que provocaban el atraso y cuáles eran los caminos que permitían salir de él”. (Baytelman, 1968).

Por su parte, los autores mencionan en la Introducción que el objetivo inicial de la investigación radicó en el interés de la CORFO por conocer la incidencia que la fragmentación de la tierra en San Pedro podría tener en la correcta implementación de un proyecto de regadío elaborado por el Ministerio de Obras Públicas. Este incluía el traslado de ciertos ayllus y un posible reagrupamiento de la propiedad fragmentada (fenómeno por el cual las unidades agrícolas de producción se encuentran compuestas por predios no contiguos) y coincidía con otros que la CORFO estaba llevando adelante en el lugar (creación de una cooperativa, construcción de un hotel y de una estación experimental). La investigación se orientó finalmente de acuerdo a objetivos más amplios, pues -desde el punto de vista de los autores- el problema de la fragmentación era apenas uno entre varios presentes en la estructura agraria local. En esencia, se procuró entender la dinámica existente entre los factores estructurales -que el medio imponía- y los culturales, debidos a las características de su población. Por lo tanto, se partía del presupuesto de que sólo el análisis conjunto del proceso productivo, el marco estructural, la población y los recursos podía explicar el estado actual de San Pedro de Atacama y ayudar a comprender la incidencia de la fragmentación en la zona.

Según el Informe, en 1961 San Pedro subsistía exclusivamente en base de una precaria economía agrícola. Sobre el total de las tierras productivas, sólo el $69 \%$ estaban en uso y, de estas, el 66,3\% se dedicaban a la alfalfa, un producto no alimenticio orientado al mercado y destinado a forraje. El cultivo de alfalfa fue introducido por los españoles para alimento mular y -a la fecha del Informe- era destinado al ganado local. En importancia decreciente seguían trigo (14,5\%), maíz $(12,24 \%)$, frutales $(2,66 \%)$ y leguminosas chañar- $(2,10 \%)$. Por su utilidad, entre los diferentes tipos de ganado se destacaban los ovinos (60\% del total), que proporcionaban carne, lana y cuero para uso doméstico; y los asnos y mulas (6,7\% del total), usados para la labranza. Estos últimos constituían un elemento de diferenciación social entre los agricultores, ya que los poseedores obtenían mayores ingresos por arriendo y por la baja de costos en la roturación de sus campos, que era la labor más costosa (Figura 3).

Los autores señalan como característica de la orientación productiva de San Pedro su "distorsión". Los frutales, con un área cultivada mínima, proporcionaban ocho veces más ingresos que la alfalfa, a la que se dedicaba más de la mitad de las tierras. Esta anomalía tenía raíces históricas. La extensa área cultivable de San Pedro, sumada a su ubicación estratégica entre el sur de Bolivia, el NOA argentino y la costa chilena, habían potenciado -particularmente durante el ciclo del salitre- la producción de forraje para mulares y bovinos, que descansaban y engordaban en la zona y luego eran llevados a los sitios extractivos. Esto había traído al pueblo un "período de esplendor" que acabó 


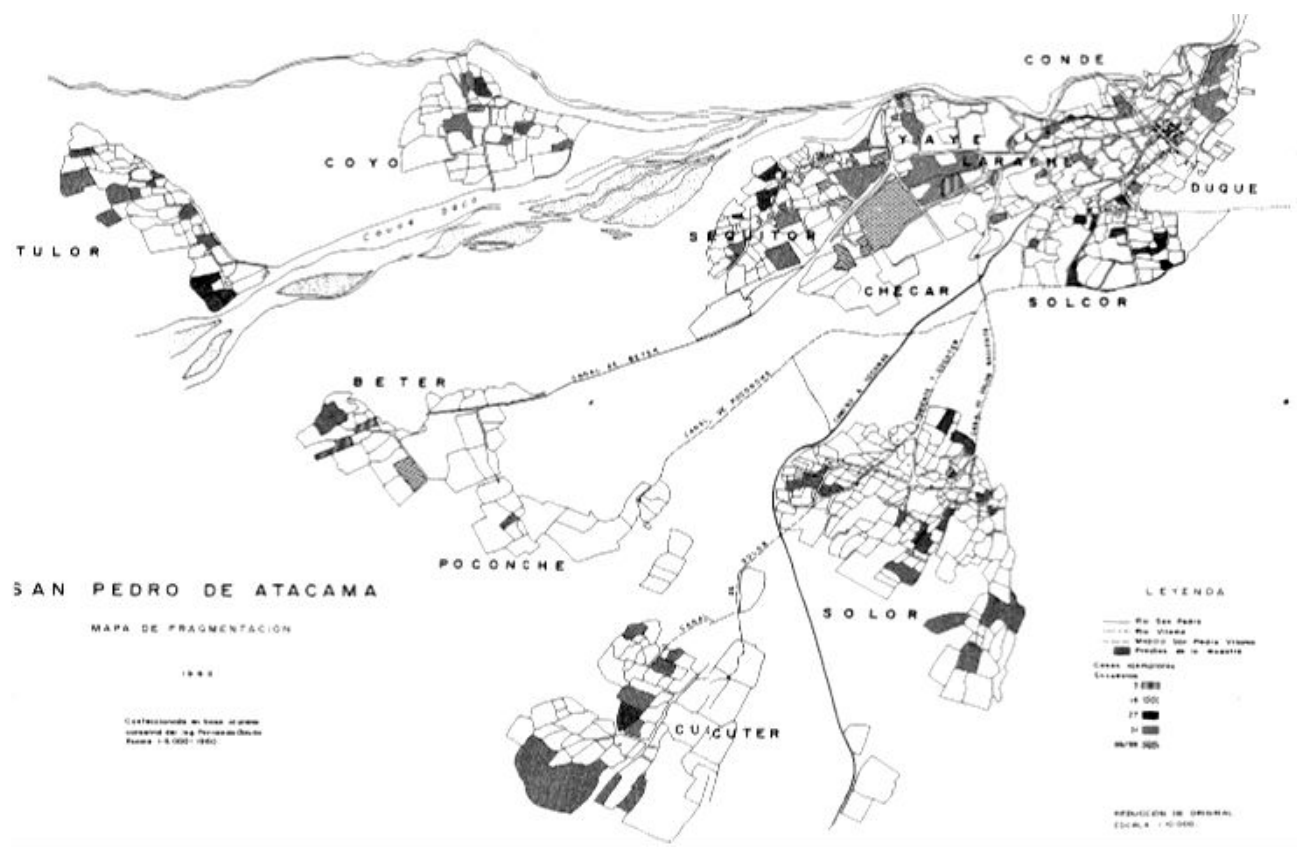

Figura 3. Mapa de fragmentación en San Pedro, tomado de Aranda et al. (1968). En diferentes tonos de gris, las áreas de los ayllus efectivamente relevadas por los investigadores

definitivamente con la construcción del ferrocarril Antofagasta-Oruro en 1917, el cual -hacia mitad del siglo XX- acaparaba ya el total del traslado del ganado. Finiquitada la arriería, se produjo una "brusca contracción de las actividades" que, sin embargo, no llevó a los agricultores a reorientar su producción (Aranda et al., 1968). Ya en 1853 -época previa al auge del salitre- Rodulfo Phillipi observó igualmente la primacía de los cultivos de alfalfa destinados al ganado mular (Phillipi, 1860). Hoy en día, cuando la agricultura sampedrina ha adquirido un carácter testimonial, reemplazada por los empleos en minería y turismo, son comunes todavía los cultivos de alfalfa (ver Figura 2). Por lo tanto, la importancia dada a este cultivo en el presente era una situación "obsoleta, derivada de un pasado en que las empastadas cumplían otra función". Así, el tipo predominante de producción era una supervivencia, testigo de un estado de cosas ahora inexistente, que los pobladores no podían cambiar debido a su pobre capitalización.

Con respecto a los intercambios, los autores abordan en primer lugar el mercado externo. Aquí se destaca que la relación entre productores y consumidores estaba plagada de intermediarios, lo que incrementaba los costos ocasionando pérdida de ingresos a los productores. La presencia de estos agentes dejaba al desnudo la ausencia de servicios y la defectuosa estructura de los canales de comercialización. Otro tipo de intercambio, que se realizaba por fuera del mercado monetario, era el trueque. La importancia de este radicaba en que otorgaba "valor de intercambio a rubros que no lo tienen en el mercado monetario [y en que además era] un índice de la unidad del área atacameña, manifestada en este caso por una integración económica hecha al nivel de un intercambio con base ecológica" (Aranda et al., 1968) entre los pastores del altiplano boliviano y los agricultores sampedrinos. Finalmente, el mercado interno era reducido y en él los almacenes tenían poder sobre los agricultores y libertad para fijar precios.

El capítulo siguiente está destinado a delinear la estructura agraria local, definida como el marco institucional que regulaba las relaciones interpersonales en cuanto al acceso a la tierra, al agua y a la fuerza de trabajo. En la época del Informe, San Pedro era una unidad territorial dividida en catorce ayllus agrupados de acuerdo al sistema de riego en tres secciones ordenadas de norte a sur, siendo los derechos de propiedad principalmente individuales. Los ayllus de las diferentes secciones eran: sección I: ayllus Catarpe, Quitor, Conde Duque, Solcor, Yaye, Larache, Sequitor, Vilama, Chécar; sección II: 


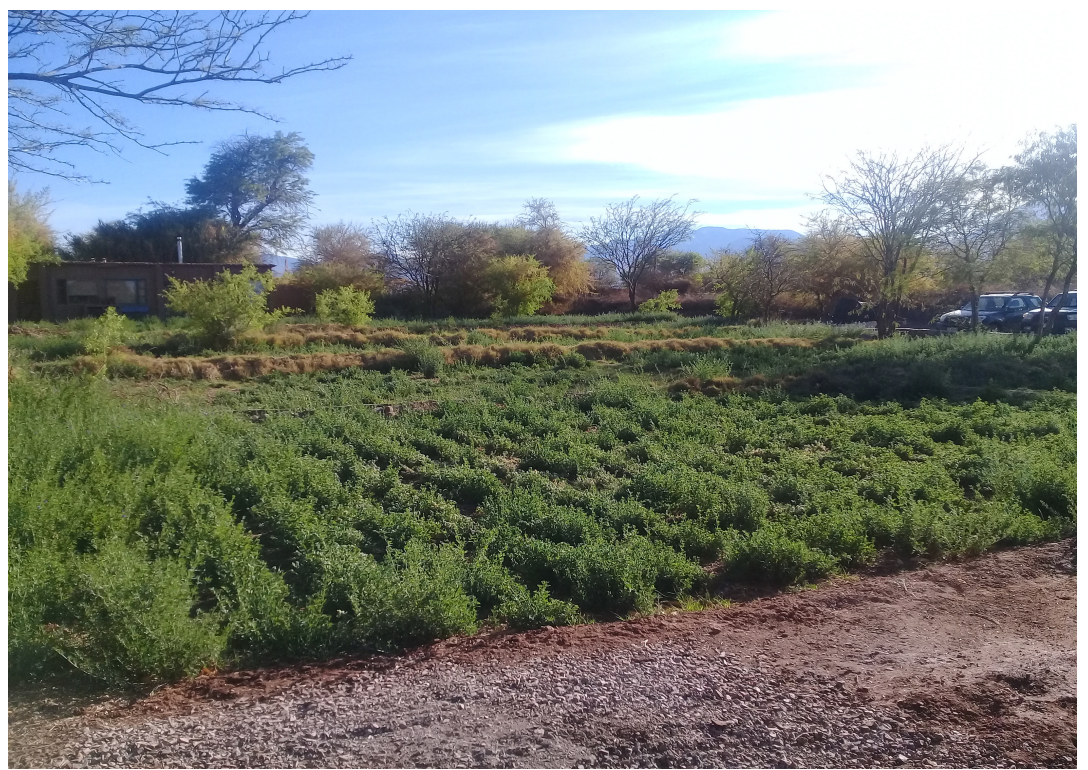

Figura 2. Cultivo de alfalfa en Solcor. Imagen testimonial de la agricultura sampedrina actual. Fotografía del autor

ayllus Sólor y Cúcuter; y sección III: ayllus Beter, Poconche, Tulor y Coyo. La subdivisión de la tierra era muy intensa -en promedio los predios tenían 1,69 hectáreas- aunque variaba entre secciones. La sección I era la más subdividida y la 3 , la menos, siendo la subdivisión inversamente proporcional al acceso al riego. Sobre un total de 1.754 hectáreas, divididas en 1.035 predios con 437 propietarios, la estructura agraria presentaba una clara concentración: el grupo de propietarios de predios mayores a 5 hectáreas poseía el $60 \%$ del total de las tierras. Esto no significaba que existiese una clase terrateniente, porque el tamaño de las propiedades no estaba en relación con la riqueza de cada familia sino con el umbral de subsistencia de cada una, por debajo del cual se hubiese visto obligada a vender su fuerza de trabajo.

Queda por tratar el tema de la fragmentación. Esta se entendía como la existencia de un área de propiedades formadas por predios no contiguos y constituía, en principio, un problema, porque-técnicamente- mientras mayor fuera esta, menor sería la eficiencia productiva. La fragmentación sampedrina se explicaba por tres factores: 1) "desaparecido el sistema comunitario (en la publicación citada, aludirán a San Pedro como una "zona de comunidades en descomposición"), fue sustituido por un régimen de propiedad privada sobre la tierra en que las leyes sucesorias han sido celosas cuidadoras de una repartición equitativa de la tierra entre los descendientes" (Aranda et al., 1968); 2) una desigual distribución en la calidad de los recursos incitaba a la tenencia de tierra en diferentes sectores; y 3 ) porque "este es un lugar que ha experimentado muchos cambios en cuanto a nivel económico que en estos momentos ha profundizado las diferencias entre los campesinos". (Aranda et al., 1968). Sin embargo, la demanda externa de fuerza de trabajo en minas y salitreras impidió que el fenómeno se intensificase, al absorber la fuerza de trabajo de la población en aumento.

La fragmentación era, pues, "moderada", y estaba "detenida", sin representar el principal de los problemas a resolver. Por lo que -a la hora de implementar futuras políticas de desarrollo- se debía cuidar de no agudizarla, toda vez que un impulso a la producción traería mayores presiones sobre la propiedad que podrían aprovechar más fácilmente los individuos mejor posicionados económicamente, acentuando de este modo la desigualdad social. Singularmente, en las encuestas realizadas, los propietarios no tenían una percepción negativa de la fragmentación ni deseaban el reagrupamiento de sus predios. Para los autores, entre otras razones de menor peso para explicar esta actitud, se encontraba una ventaja del sistema tradicional: su versatilidad, en tanto la fragmentación permitía la separación de tareas, de cultivos y de animales. 
En el capítulo 3 se analiza la población y los problemas de la fuerza de trabajo. El censo de 1960 indicaba una cantidad de 819 personas, habiendo disminuido un $35 \%$ desde 1952. Esta baja era común a todas las localidades de agricultura tradicional del Norte Grande y su contraparte era el aumento poblacional de los centros urbanos importantes (como Calama, Antofagasta y Salta). La emigración daba como resultado no sólo una merma sino también un envejecimiento de la población, porque los emigrantes se concentraban en el segmento de los 20/40 años. La gente migraba por la contracción de la economía local y por la atracción que ejercían los sectores más dinámicos de la economía regional: los salarios más elevados de la minería en Chuquicamata o los múltiples atractivos que las urbes ofrecían. Finalmente, se consideraba que el fenómeno no era de carácter estacional, sino que generalmente implicaba la radicación en los lugares de destino.

En cuanto a las características y demanda de mano de obra familiar y ajena, la ganadería precisaba de atención sostenida el año entero, mientras que la agricultura exigía poca mano de obra y se concentraba en determinadas épocas. La limpieza de canales y algunas labores agrícolas eran las únicas actividades para las cuales se vendía la fuerza de trabajo, ya que la mayor parte de las tareas recaía sobre los mismos dueños y su grupo familiar. Un elemento que sobresalía era el intercambio de mano de obra o minga, institución que -aunque al momento del informe cobraba fuerza por el poco metálico circulante- tenía profundas raíces históricas. La minga se realizaba en tiempos de siembra y de cosecha y asistían a ella hombres, mujeres y niños. Los hombres realizaban las tareas específicas, las mujeres preparaban la comida y los niños hacían pequeñas labores o permanecían como espectadores. En la minga participaban parientes y amigos con sus herramientas. Estos eran convocados por el dueño del predio y, de acudir, generaban la obligación de "devolver la mano". Por fuera de la minga, las tareas diarias anuales se hacían en base de una división sexual del trabajo: las mujeres estaban vinculadas a las tareas más tradicionales y que implicaban cierta ceremonialización (maíz, algarrobo, ganadería) y los hombres a las tareas más modernas (alfalfa y trigo).

El capítulo 4 describe con detalle los recursos y las técnicas de cultivo. Los recursos hídricos eran aportados por los ríos San Pedro y Vilama y distribuidos por una red de canales y acequias que llevaba el agua a cada uno de los ayllus, guardándose un estricto orden de turnos.
En este sistema, que favorecía sin distinción a todos los pobladores, como en la participación obligatoria de los beneficiarios en los trabajos comunes y en la responsabilidad y vigilancia de este valioso recurso que se expresaba también en instituciones específicas -como la Junta de Riego, el Inspector de Aguas y el Reglamento de Riego- los autores observaban "la persistencia de modalidades relictas de un pasado comunitario no muy lejano" (Aranda et al., 1968). Para profundizar en esta temática desde una perspectiva agronómica, histórica y social, véase Sepúlveda Rivera et al. (2015).

Con base en el anterior diagnóstico, el Informe proponía un plan de ayuda crediticia y técnica a los productores directos. Este debería tomar en cuenta las instituciones propias de la comunidad para que esta "desarrolle los planes como un todo y no como productores aislados entre los cuales surjan diferencias marcadas de ingresos". (Aranda et al., 1968: 150). Se visualizaba la coexistencia de dos formas de propiedad. La familiar, mejorada mediante medidas que detuviesen la fragmentación, y la cooperativa, "formada por un fondo de tierras en que el aporte sea mano de obra y no dinero [y] que reuniría [...] toda la superficie en una unidad". (Aranda et al., 1968). En tanto los autores caracterizan a San Pedro como una "zona de comunidades en descomposición" (Aranda et al., 1968), debido al proceso de privatización de la tierra y por la emigración, la cooperativa tendría un basamento compatible con cierta tradición de los ayllus atacameños (Figura 4).

\section{Conclusiones}

En definitiva, el Informe constituye una investigación fundamentada sobre los distintos elementos que se deberían tomar en cuenta a la hora de implementar planes de desarrollo, proponiéndose que estos incorporen no solo inversiones estatales y know-how de profesionales, sino que también aprovechen las condiciones locales (Aranda et al., 1968: ii). El Informe se basa en un detallado análisis de los recursos naturales y humanos, del proceso productivo y del marco estructural de la producción, para dar cuenta -con una mirada que es estructural e histórica a la vez- del estado de la producción del área. La investigación relevó además un conjunto de prácticas que demostraban la persistencia de formas de intercambio de raíces precolombinas, como la arriería que conectaba diferentes pisos ecológicos -pastores altiplánicos y agricultores del valle- o la 


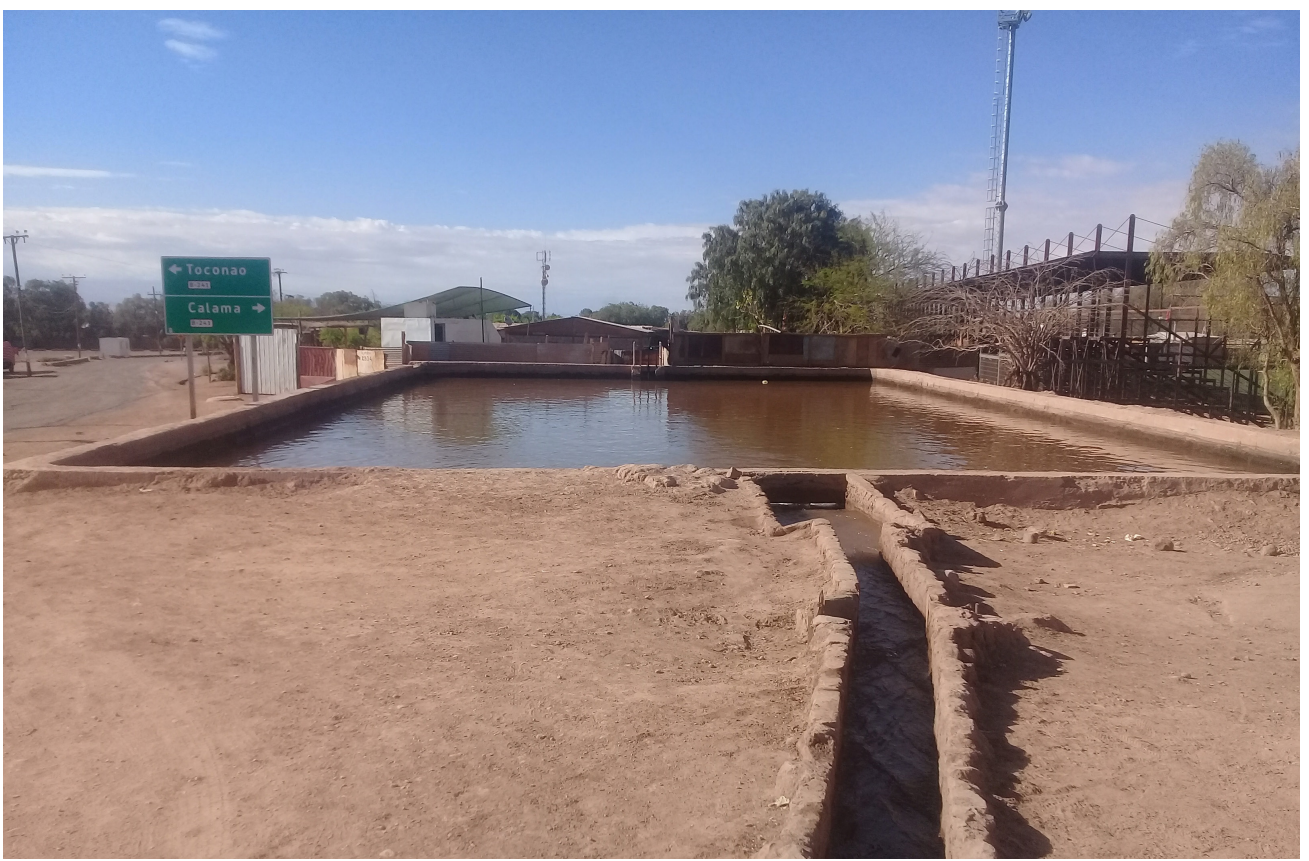

Figura 4. Reservorio de agua -ubicado a pocos metros del centro de San Pedro- que forma parte del importante sistema de regadío que surte los espacios agrícolas. Fotografía del autor

minga, consistente en contraprestaciones de trabajo comunitario. Y, a la vez, resaltó que en el presente estas mismas prácticas se combinaban con otras más modernas, como la minga con el trabajo asalariado.

El Informe impacta por la calidad de los datos aportados en una época en que las investigaciones en el área estaban en sus comienzos, constituyendo una obra pionera sobre temas que luego estarían en la mira de los estudios andinos. Encontramos así tempranas problematizaciones sobre el logro andino (sensu Murra, 1972), ya que -en la evaluación final- el problema de la fragmentación constituía una respuesta propiamente andina (tradicional y moderna a la vez) a la multiplicidad de factores que incidían en la agricultura local.

Por otro lado, no debemos obviar un elemento de importancia que los autores -tal vez por lo relativamente moderno del proceso, tal vez por el enfoque adoptado- no pudieron ver. Se trata de lo que hoy conocemos como postcomunalidad, proceso iniciado a mediados del siglo XX alentado por cambios en la economía regional y que incluyó desruralización (por emigraciones y movilidad), desagrarización (del patrón de ingresos), descampesinización (de la organización económica) y translocalización (de los patrones de relaciones sociales) de las poblaciones andinas (Gundermann Kröll y González Cortez, 2008). Estos cambios en los patrones de movilidad, asentamiento y relatedness de la población indígena llevaron a que en el presente asistamos a una regionalización consistente en la formación de un sistema de relaciones desplegado entre las localidades andinas y las áreas de migración, en ambos sentidos (Gundermann Kröll, 2001). Desde esta perspectiva, entonces, el movimiento poblacional descrito en forma negativa en el Informe debería ser entendido como parte de un proceso más general que no condujo inevitablemente a la descomposición de las comunidades sino a su resignificación en clave regional.

En perspectiva, se puede decir que, aunque el Informe es un ejemplo claro de la matriz intelectual de su época-las ciencias sociales puestas al servicio de las políticas desarrollistas-, sobresale también por la complejidad de su mirada, que evita quedar encorsetada en el puro tecnicismo. Por lo tanto, investigaciones como la tratada en este artículo impulsaron no solo el conocimiento sobre el tema propio de pesquisa -la cuestión agraria- sino que también contribuyeron significativamente al entendimiento de realidades antropológicas específicas. 


\section{Literatura citada}

Aranda, X.; Barahona, R.; Saá, R.

1968. Elementos diagnósticos para un plan de desarrollo local en San Pedro de Atacama. CORFO-IG-UCH. Santiago de Chile, Chile. 226 p.

Baytelman, D.

1968. Introducción. En: Aranda, X.; Barahona, R. y Saá, R. Elementos diagnósticos para un plan de desarrollo local en San Pedro de Atacama: iv-vi. CORFO-IG-UCH. Santiago de Chile, Chile. pp. 1-3.

Chiappe, C.

2015. Los estudios andinos, entre la reforma y la revolución. Crear-El Jote Errante. Iquique, Chile. 188 p.

Chiappe, C.

2018. Transformaciones agronómicas en el Valle de Azapa. Una etnografía pionera de Tristan Platt (1975). IDESIA, 35 (3): 41-49.

Consejo de Dirección Prismas.

2007. Presentación. Prismas. Revista de historia intelectual, 11: 151.

Galdames Rosas, L.; Ruz Zagal, R.

2010. La Junta de Adelanto de Arica y John V. Murra. Dos lecturas sobre el desarrollo andino en el norte de Chile. Chungara, Revista de Antropología Chilena, 42 (1): 257-270.

Garretón, M.

2005. Social Sciences and society in Chile: institutionalization, breakdown and rebirth in Chile. Social Sciences in Latin America. 44p.

Gundermann Kröll, H.

2001. Procesos regionales y poblaciones indígenas en el norte de Chile. Un esquema de análisis con base en la continuidad y los cambios de la comunidad andina. Estudios Atacameños. Arqueología y antropología surandinas, 21: 89-112.

Gundermann Kröll, H.; González Cortez, H.

2008. Pautas integración regional, migración, movilidad y redes sociales en los pueblos indígenas de Chile. Universum, 23 (1): 82-115.

Gundermann Kröll, H.; González Cortez, H.

2009. Sociedades indígenas y conocimiento antropológico. Aymaras y atacameños de los siglos XIX y XX. Chungara. Revista de Antropología Chilena, 41 (1): 113-164.

Kuhn, T.

2005. La estructura de las revoluciones científicas. Fondo de Cultura Económica Madrid, España. 361 p.
Murra, J.

1972. El 'control vertical' de un máximo de pisos ecológicos en la economía de las sociedades andinas. En: Murra, J. Visita de la Provincia de León de Huánuco en 1562, Vol. 2. Universidad Hermilio Valdizán. Huánuco, Perú. pp. 429-476.

Núñez, L.

2010. De las apariciones y andanzas de John Murra por el desierto de Atacama y cómo construyó su misión innovadora. Chungara, Revista de Antropología Chilena, 42 (1): 127-139.

Núñez, L.

2013. Sobre los comienzos de los estudios andinos y sus avances actuales en el norte de Chile. En: Zanolli, C. (comp.). Los estudios andinos hoy. Práctica intelectual y estrategias de investigación. Prohistoria. Rosario, Argentina. pp. 79-122.

Pérez Rodríguez, E.

1984. Políticas de desarrollo en la zona del Interior y Altiplano: Tarapacá-Chile. Cuadernos de Investigación Social 9. Iquique, Chile. 58p.

Phillipi, R.

1860. Viaje al desierto de Atacama: hecho de orden del gobierno de Chile en el verano de 1853-54. Eduardo Antón. Halle, Sajonia. 236 p.

Rivera, M.

1973a. A manera de introducción: Hacia una política de desarrollo integral del Departamento de Arica. Chungara, Revista de Antropología Chilena, 1-2: 11-16.

Rivera, M.

1973b. Bases para planificar la investigación antropológicaarqueológica en el Norte Grande Chileno. Chungara, Revista de Antropología Chilena 1-2: 39-48.

Sepúlveda Rivera, I.; Molina Otárola, R.; Delgado-Serrano, M.; Guerrero Ginel, E.

2015. Aguas, riego y cultivos: cambios y permanencias en los ayllus de San Pedro de Atacama. Estudios Atacameños. Arqueología y antropología surandinas, 51: 185-206.

Taberna Gallegos, F.

[1973] 1996. Simposio $N^{\circ}$ 4: El rol de la sociedad andina y el tránsito al socialismo. Informe: Algunas Condiciones Básicas para el estudio del tránsito hacia el Socialismo de la Sociedad Andina. En: Guerrero, B. (ed.) Homenaje a Freddy Taberna Gallegos. Escritos sobre la sociedad aymara del Norte Grande de Chile. CREAR-El Jote Errante. Iquique, Chile. pp. 47-65. 